\title{
syn-1,2-Amino Alcohols via Allylic C-H Amination
}

Metal-Catalyzed

Asymmetric

Synthesis and

Stereoselective

Reactions

\section{Key words}

amino alcohols

C-H amination

palladium

SYNFACTInth
Significance: Amino alcohols are unquestionably important compounds in biologically relevant settings. This paper describes a new approach for the diastereoselective synthesis of these compounds starting from a homoallylic alcohol derivative. Palladium catalyzed allylic $\mathrm{C}-\mathrm{H}$ amination provides these important molecules in an expedient and selective manner.
Comment: The relatively high selectivity for this new process as well as the functionality of the product (alcohol, amine, and olefin) make this protocol extremely interesting. The White group has found quite a number of interesting uses for the $\mathrm{Pd}(\mathrm{II})$-sulfoxide complex system. Allylic $\mathrm{C}-\mathrm{H}$ functionalization seems to be a general trend, making this catalyst class quite intriguing for future exploitation. 